\title{
Wet chemical deposition of transparent conducting coatings in glass tubes
}

\author{
J. Puetz*, F.N. Chalvet, M.A. Aegerter \\ Institut für Neue Materialien, Coating Technology, D-66123 Saarbruecken, Germany
}

\begin{abstract}
A modified dip coating process was developed to deposit transparent conducting sol-gel $\mathrm{SnO}_{2}$ :Sb (ATO, antimony doped tin oxide) coatings inside tubes and cavities in optical quality. The necessity for this improvement arises from the fact that the conventional dip coating process applied to tubes results in coatings with poor and non-reproducible properties, showing large variations in the thickness, an increased roughness and an island-like morphology. The main reason for this is found in a delayed and restricted solvent evaporation, due to an impediment of a laminar flow and a progressive saturation of the atmosphere inside the tube. By means of an additional exhausting pipe, a forced laminar flow was, therefore, generated inside the tube, which guarantees a fast evaporation and hydrolysis of the sol-gel films by a continuous renewal of the atmosphere. In this way, ATO coatings with an excellent thickness uniformity and a low roughness of $R_{\mathrm{a}} \approx 1 \mathrm{~nm}$ could be deposited on borosilicate glass tubes of $300 \mathrm{~mm}$ length and inner diameters down to $11 \mathrm{~mm}$. After a heat treatment at temperatures up to $550{ }^{\circ} \mathrm{C}$, single layer coatings exhibit a high optical transmission $(T>85 \%)$ and a resistance down to $10 \mathrm{k} \Omega$ over a length of $22 \mathrm{~cm}$.
\end{abstract}

Keywords: Sol-gel process; Electrical properties and measurements; Optical coatings; Tin oxide

\section{Introduction}

Transparent conducting oxide (TCO) coatings combine the property of a high optical transmission with that of a high electrical conductivity, which makes them an essential component in a large variety of applications $[1,2]$. Coating materials like ITO (tin doped indium oxide, $\mathrm{In}_{2} \mathrm{O}_{3}: \mathrm{Sn}$ ) and ATO (antimony doped tin oxide, $\mathrm{SnO}_{2}: \mathrm{Sb}$ ) can, for example, act as electrodes (e.g. in liquid crystal displays, solar cells) or give antistatic properties to glasses or plastics (e.g. on cathode ray tubes). In most cases physical or chemical vapour deposition techniques (PVD, CVD) are used to deposit such TCO coatings [3] as they result in a low sheet resistance (typically $10 \Omega_{\square}$ ) and guarantee a high optical quality as it is required especially for displays and solar cells. However, the corresponding techniques are optimised to coat flat or weakly bent substrates whereas complex surfaces like the inner surfaces of tubes and cavities are not accessible or accessible only after extensive adaptations. Examples for such highly specific coating techniques for the inside coating are the

*Corresponding author. Tel.: +49-681-9300-349; fax: +49-6819300-249.

E-mail address: puetz@inm-gmbh.de (J. Puetz). pulsed laser deposition (PLD), where a target is evaporated inside the tube by means of a laser [4], ion beam sputtering [5] or plasma CVD [6].

It would, therefore, be of advantage to have a low cost and flexible deposition technique at hand, as it is found in the liquid film coating [7]. The dip coating technique, which is known for the high uniformity of the resulting coatings, has already been described to deposit lacquer systems on tubes with several suggestions for improvements and modifications [8-10]. However, sol-gel coatings have not been deposited so far inside tubes by dip coating, though this technique offers a convenient and low-cost alternative to obtain high quality coatings.

\section{Experimental}

\subsection{Film preparation and characterization}

Sol-gel ATO coatings were prepared by the dip coating technique using an $0.2 \mathrm{M}$ solution of $\mathrm{SnCl}_{2}$ in ethanol with a doping of $5 \mathrm{~mol} \% \mathrm{SbCl}_{3}$ and $0.4 \mathrm{M}(2$ equiv.) diacetone alcohol (4-hydroxy-4-methyl-2-pentanone). The obtained solution was stirred under reflux for $1 \mathrm{~h}$ and filtered through a $0.2 \mu \mathrm{m}$-PTFE filter. The 
coatings were deposited with a constant withdrawal speed of $4 \mathrm{~mm} / \mathrm{s}$ on borosilicate glass tubes (Duran, Schott Rohrglas) with a length of $300 \mathrm{~mm}$ and outer diameters of $14 \mathrm{~mm}(1.5 \mathrm{~mm}$ thickness $)$ and $50 \mathrm{~mm}$ (1.8 $\mathrm{mm}$ thickness), respectively. During the deposition the atmospheric conditions were kept constant at $(21 \pm 1){ }^{\circ} \mathrm{C}$ and $(40 \pm 2) \%$ RH. After a hydrolysis time of $1 \mathrm{~min}$, the as-deposited films were dried at $250{ }^{\circ} \mathrm{C}$ (15 min) and subsequently heat treated with a heating rate of $2.5 \mathrm{~K} / \mathrm{min}$ in a convection furnace in air at 550 ${ }^{\circ} \mathrm{C}(15 \mathrm{~min})$ to form the desired ATO coatings. Additional coating experiments were carried out to investigate the evaporation behaviour of the deposited wet films on the tubes during dip coating by means of a thermographical camera (Thermovision 400, Agema) using pure ethanol.

For a characterization of the coatings, the tubes were cut into pieces of $20 \mathrm{~mm}$ or $43 \mathrm{~mm}$ length, respectively. After rinsing with water and drying, the sheet resistance $R_{\square}$ was then determined by the four-point technique using Au tips in a linear arrangement (34401 A, Hewlett-Packard). For the same samples the coating thickness $d$ was measured by surface profilometry (Tencor P10) after preparation of a sharp edge by chemical etching with $\mathrm{HCl}$ and $\mathrm{Zn}$ dust. Similarly, the average roughness $R_{\mathrm{a}}$ of the coatings was obtained from a measurement with the same device over a distance of $0.5 \mathrm{~mm}$. The film morphology was investigated by scanning electron microscopy (SEM) (15 keV, JSM $6400 \mathrm{~F}$, Jeol) after deposition of a thin Au layer on top of the film.

\subsection{Modified dip coating process}

A modified dip coating apparatus was developed, as shown in Fig. 1, in order to obtain a forced laminar flow inside the tube. This was accomplished by an additional exhausting glass pipe $(5 \mathrm{~mm}$ outer $\varnothing)$ that was placed in the solution vessel and introduced from below into the tube with the internal end rising $5 \mathrm{~mm}$ above the solution. This arrangement brings almost no restrictions compared to the conventional process with regards to deposition speed and handling. The external end of the exhausting pipe was connected to a diaphragm vacuum pump with an adjustable, constant flow of air of up to $210 \mathrm{l} / \mathrm{h}$. The flow of air that is necessary to obtain homogeneous films depends on the withdrawal speed, on the inner diameter of the tube and also on the coating solution so that an optimisation is necessary. For the two tubes used in these experiments a constant flow of $50 \mathrm{l} / \mathrm{h}$ in the case of the $14 \mathrm{~mm}$ tubes and $210 \mathrm{l} / \mathrm{h}$ for the $50 \mathrm{~mm}$ tubes was generated.

For the formation of a constant laminar flow through the tube, it is important that the lower end of the tube remains in the solution in order to enclose the exhausting pipe and force the air to go through the tube. It is,

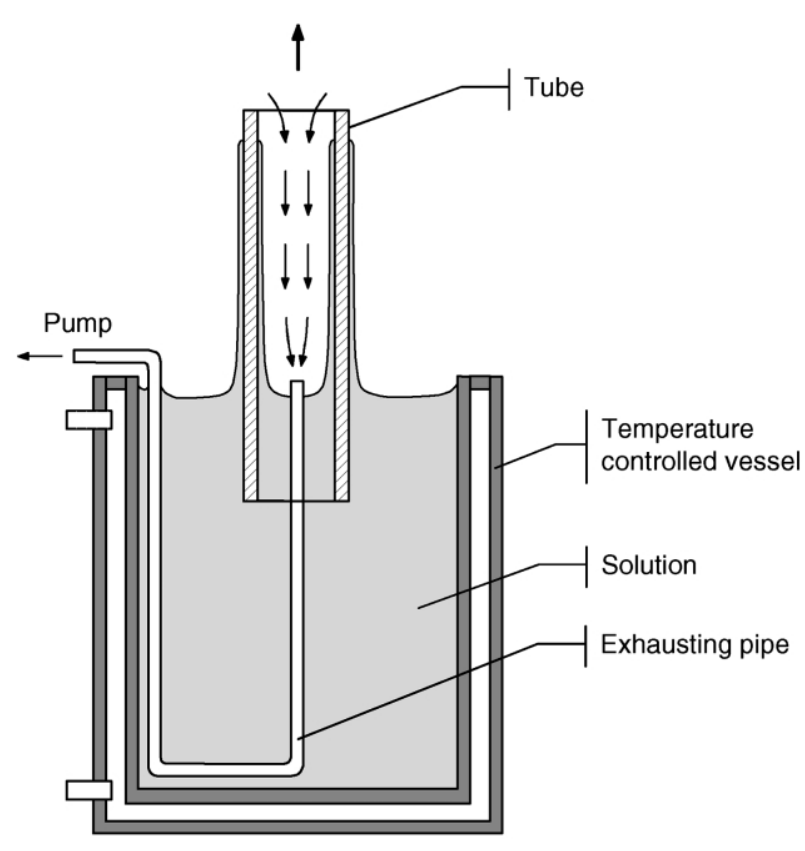

Fig. 1. Experimental set-up for the dip coating of tubes with an internal exhausting pipe and a temperature controlled vessel.

therefore, necessary to modify the sequence of the dip coating process compared to the coating of flat substrates, as on complete withdrawal the exhausting pipe would be bared and the internal flow would come to an end. Thus, the tube is not completely withdrawn but is kept in the solution just enough to seal the tube in the lower end. After a drying time of 1 min the substrate tube is then removed from the solution and the adhering solution is wiped off. The thus prepared films subsequently were dried and heat treated as described before.

\section{Results and discussion}

\subsection{Conventional dip coating process}

The dip coating process is the most often used deposition technique for sol-gel thin films on flat substrates because of the high homogeneity and uniformity of the obtained coatings. However, if the same technique is applied to tubes and cavities, the optical quality and the uniformity of the coatings are not satisfying anymore. Especially for the inner coating, basic properties like the thickness and the average roughness are not reproducible and cannot be controlled. Correspondingly, the electrical properties like the sheet resistance are poor and show large fluctuations over the tube length. This is demonstrated in Fig. 2a where the coating thickness is shown for a tube with an inner diameter of $11 \mathrm{~mm}$. The thickness variations and the large standard deviations clearly indicate that the extraordinary thickness uniformity of the sol-gel dip coating process with variations of only some nanometers 
(a)

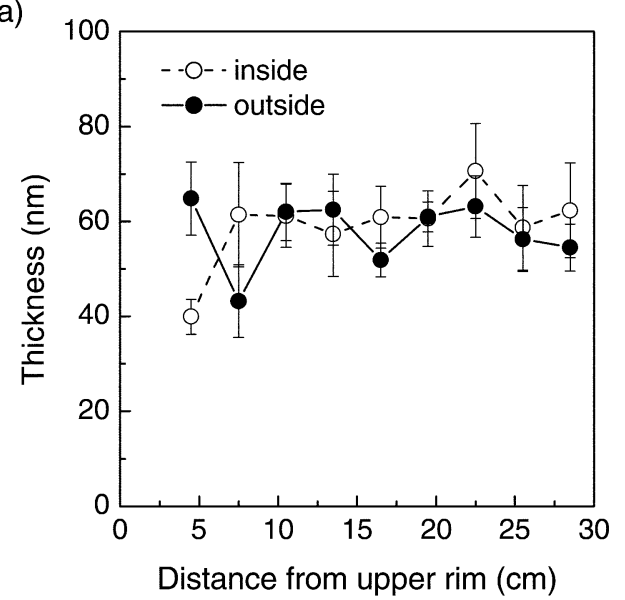

(b)

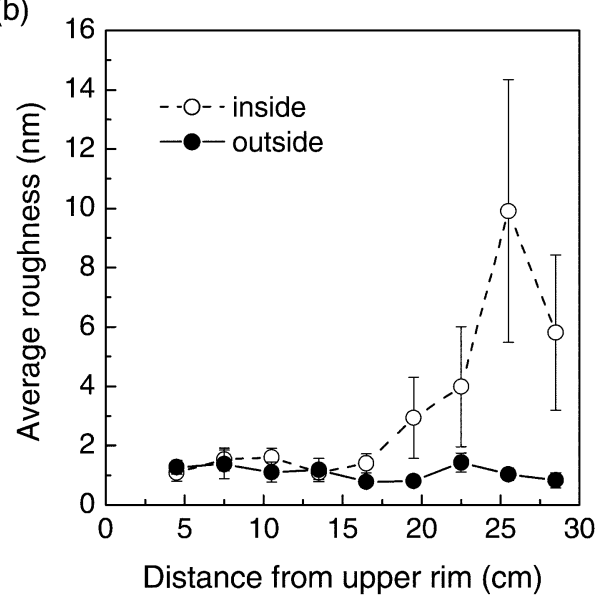

Fig. 2. Thickness $d$ and average roughness $R_{\mathrm{a}}$ of conventionally dip coated ATO coatings on tubes with an inner diameter of $11 \mathrm{~mm}$.

is not achieved for the tubes, neither inside nor outside. Nevertheless, the average thickness is comparable to the coating thickness of $(58 \pm 2) \mathrm{nm}$ obtained on flat substrates with the same parameters. The surface roughness (Fig. 2b) is much higher than expected for sol-gel films where values below $1 \mathrm{~nm}$ can be realized. Especially the inner coating exhibits an inhomogeneous distribution in the average roughness $R_{\mathrm{a}}$ with values of approximately $2 \mathrm{~nm}$ in the upper part, but up to $(10 \pm 4) \mathrm{nm}$ in the lower part of the tube. In contrast, the average roughness of the outer coating is only 1-2 $\mathrm{nm}$ and shows lower fluctuations. Similar results with regards to the thickness and the roughness were obtained for tubes with a larger inner diameter, but the fluctuations are less distinct in this case.

The coating morphology, as investigated by SEM images (Fig. 3a,c), reveals fundamental differences compared to coatings on flat substrates, which exhibit a homogeneous and coherent coating morphology (Fig. $3 e)$. On both sides of the tube, irregular structures with weak rises and deep cracks of up to $0.5 \mu \mathrm{m}$ length are found, especially for the inner coating with a low density and an almost continuous network of cracks. (Fig. 3b,d will be discussed in Section 3.2.)

Given this morphology, it is understandable that the macroscopic electrical properties of the ATO coatings thus prepared on the tubes are inferior to those on flat substrates. Due to the extreme sensitivity of the sheet resistance $R_{\square}$ to any morphological changes, both the large variations over the tube length and the porous microstructure make it impossible to obtain reproducible highly conducting coatings.

To elucidate the reasons for the different coating properties on flat substrates and tubes, a thermographical analysis of the dip coating process of the tubes was performed. The evaluation of these images is based on the fact that the temperature of the substrates decreases due to the evaporation cooling during the evaporation of the solvent. The obtained images are shown in Fig. 4a for different stages of the withdrawal process, with $t=0 \mathrm{~s}$ denoting the beginning of the withdrawal process. For a correct assignment of the temperature scale it should be mentioned that the temperature changes of the tubes are only between 16 and $20{ }^{\circ} \mathrm{C}$. In the first two images (30 and $60 \mathrm{~s}$ ), where the tube is withdrawn from the solution (ethanol), a very weak temperature decrease at the upper end of the tube is detected. The drying is initiated only when the tube leaves the solution (90 s, Fig. 4a) in a narrow area with a local minimum temperature of approximately $16{ }^{\circ} \mathrm{C}$ which is moving slowly downwards. It takes approximately 3-4 min to completely evaporate the solvent, as indicated by the subsequently rising substrate temperature. This has to be compared with the drying on flat substrates, that starts immediately after withdrawal and is completed after only $30 \mathrm{~s}$ [11]. (Fig. 4b is discussed in Section 3).

For some coating solutions, especially those containing diacetone alcohol, this local drying zone is accompanied by a hazy 'ring' (Fig. 5). A closer inspection reveals small droplets in this zone, which leads to the conclusion that the ring is either formed by a condensation reaction due to the temperature decrease or by a wetting problem. The involvement of high boiling components like diacetone alcohol further speaks for a film separation caused by the selective evaporation of volatile ethanol leading to an enrichment in the less volatile diacetone alcohol, which, however, shows a poor wetting of the glass substrates thus forming the observed droplets.

The bad coating quality, therefore, seems to originate from a saturation of the inside by the solvent vapour, in consequence leading to a restricted and delayed drying of the deposited wet film. For the initial drying and hydrolysis during sol-gel dip coating, the flow of air in 
(a)

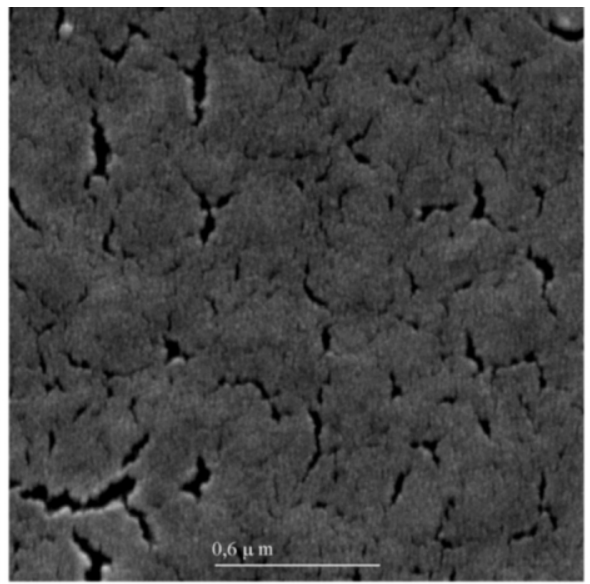

(c)

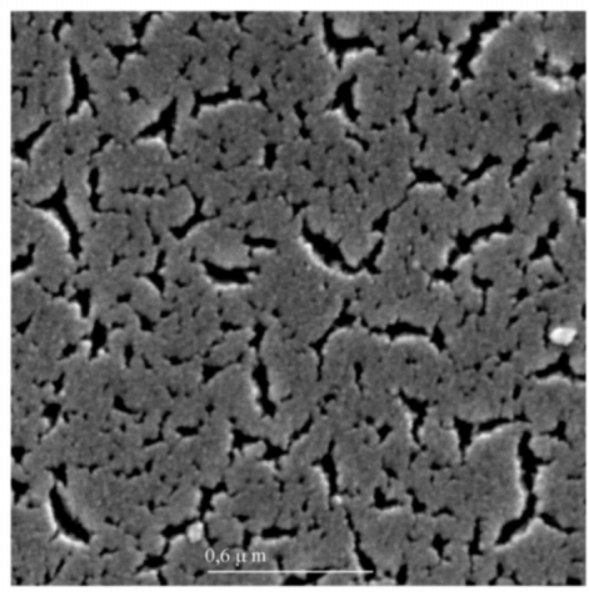

(b)

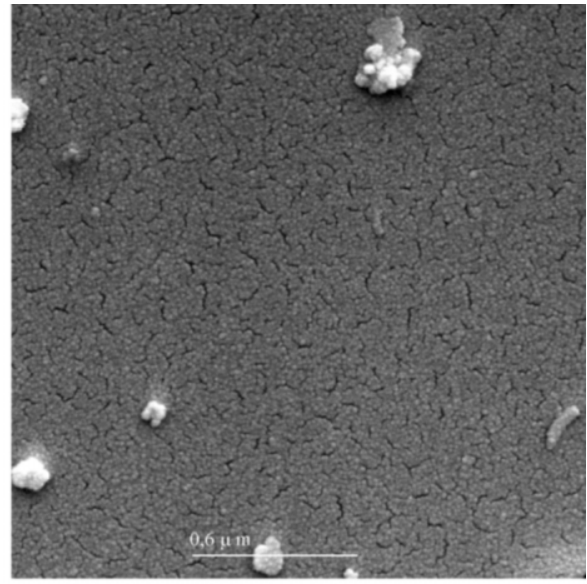

(d)

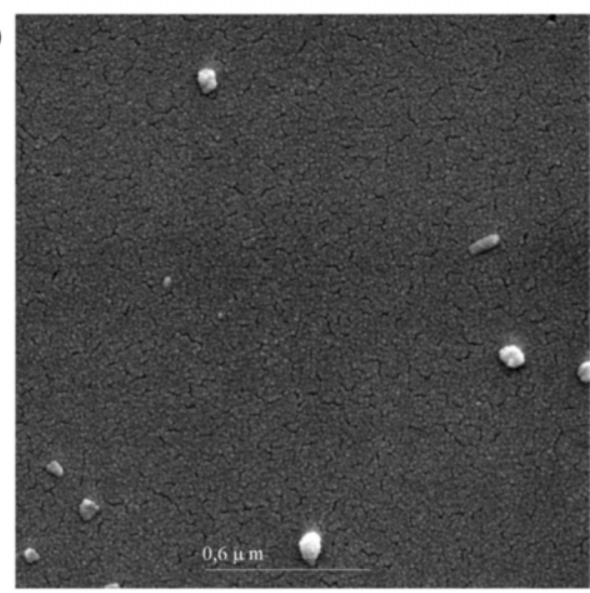

(e) Flat substrate

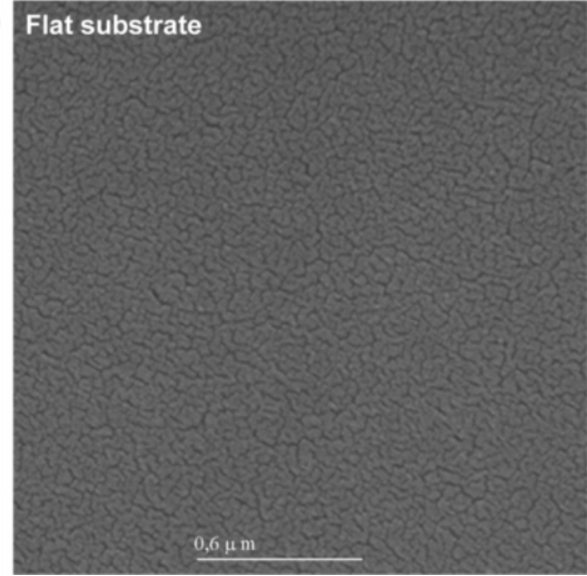

Fig. 3. SEM images of ATO coatings deposited by conventional dip coating (left) and under forced flow conditions (right) on the outer (upper) and inner side (middle) of glass tubes (inner diameter $11 \mathrm{~mm}$ ) and on flat substrates (lower).

the next surroundings of the wet film is extremely important and decisively influences the properties of the resulting coating. As the solvent vapours are specifically heavier than the ambient atmosphere, they start sinking downwards and thus generate a constant laminar flow over the film surface as it is illustrated in Fig. 6a for flat substrates. This increases the evaporation rate by reducing the partial pressure of solvent next to the surface and keeps the concentration of water constant by permanently renewing the atmosphere. For a uniform thickness, this flow has to be laminar and thus should be protected against external influences like draft and convection.

In the case of the tubes, however, this flow can only form on the outside (Fig. 6b), whereas the inside of the tubes is closed at the bottom as long as the tube is in 
the solution. Formed solvent vapours hence accumulate at the bottom of the tube and a similar flow thus is not possible. The upper part of the film, however, is almost unaffected if the withdrawal speed is not too low, as the vapours sink down in the tube and the internal volume is permanently increased during withdrawal. But especially in the lower part of the tube the evaporation is completely delayed as long as the tube is in the coating solution. Only when the tube leaves the solution a weak flow can form gradually. With decreasing inner diameter of the tubes these difficulties become more and more decisive as the inner volume is decreasing and the drag force is increasing, thus slowing down the flow rate. Another problem arising from the closed inner volume is that the content of water is limited and thus the required hydrolysis of the sol-gel materials is restricted. But it is not only the inner film that is affected by these processes, as the substrate temperature changes inhomogeneously as shown in the thermographical analysis (Fig. 4a), which also influences the drying of the wet film on the outer side.

\subsection{Forced flow conditions}

For an improvement of the coating quality, hence a saturation of the inner atmosphere with solvent has to
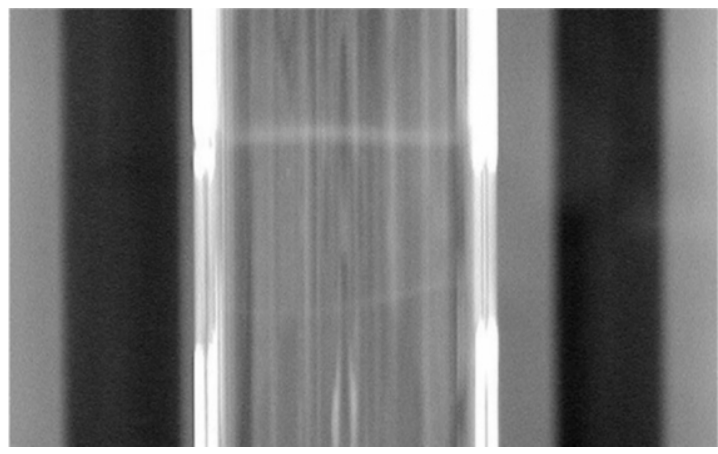

Fig. 5. Formation of a ring inside a tube during dip coating from a solution with a high boiling point component.

be avoided by forcing laminar flow conditions inside the tube. This was achieved by the use of an additional exhausting pipe that is advantageously introduced from below into the substrate tube (Fig. 1). In this way, a continuous flow of air is generated that guarantees the removal of the evaporated solvent while keeping the evaporation rate high, but that also maintains the supply of water for hydrolysis of the sol-gel wet film. It turned out that the resulting forced flow conditions are comparable to the intrinsic laminar flows that form over the outer side of the tubes or over flat substrates.

(a)
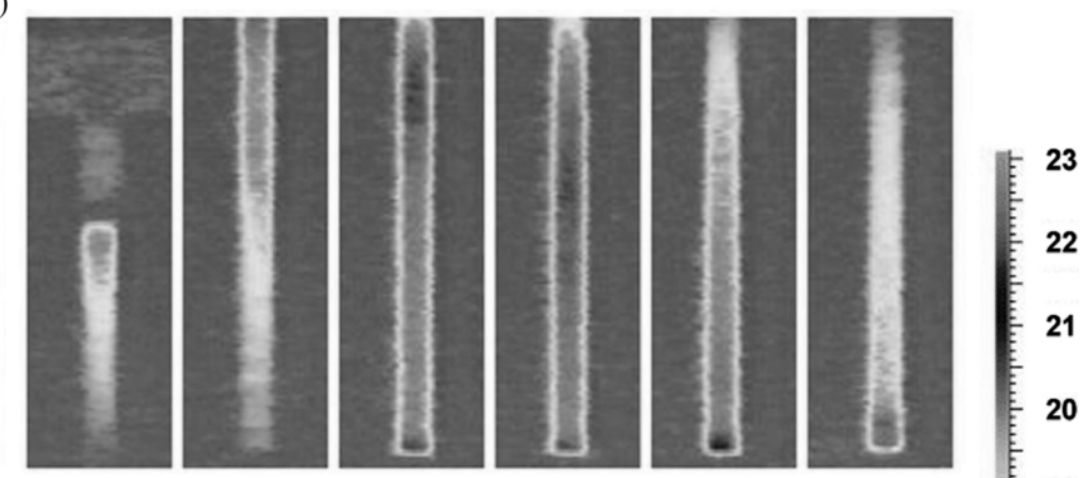

(b)
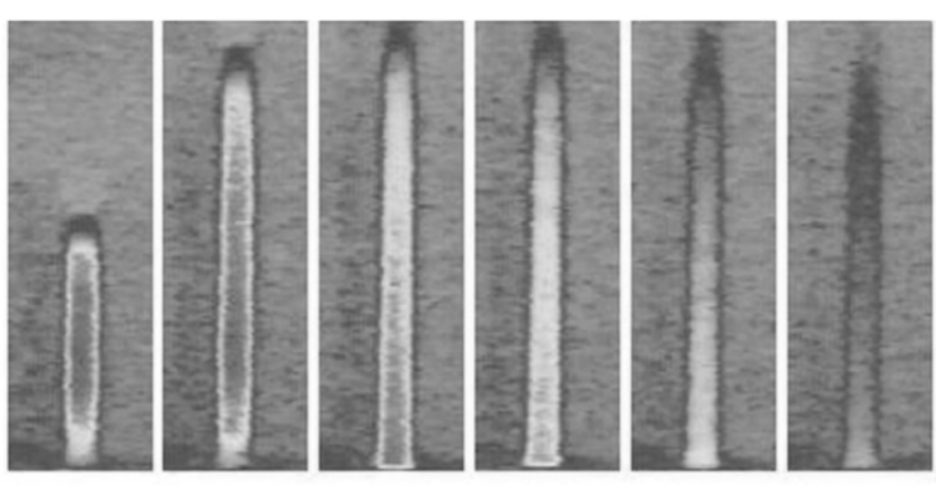

$30 \mathrm{~s}$

$60 \mathrm{~s}$

$90 \mathrm{~s}$

$120 \mathrm{~s}$

$180 \mathrm{~s}$

$240 \mathrm{~s}$

Fig. 4. Thermographical images of a borosilicate glass tube (inner diameter $11 \mathrm{~mm}$ ) during (a) conventional dip coating and (b) under forced flow conditions. $t=0 \mathrm{~s}$ corresponds to the beginning withdrawal. 
The improved drying behaviour compared to the conventional dip coating is clearly demonstrated by the thermographical analysis of the coating process (Fig. $4 \mathrm{~b})$. The solvent evaporation here starts immediately with the withdrawal process as indicated by the homogeneous temperature lowering over the whole length in the first two images of Fig. 4b. The total drying process now takes less than $60 \mathrm{~s}$ with a minimum temperature of only $18{ }^{\circ} \mathrm{C}$. The solvent evaporation hence is more homogeneous and much faster than in the case of the conventional dip coating process.

The coatings that are obtained under such forced flow conditions correspondingly show a significantly improved thickness uniformity and smoothness (Fig. 7). For both tube diameters the thickness is almost uniform over the whole length of the tube (Fig. 7a,b) with a similar average thickness of 58-62 nm. In the case of the tube with the lower diameter, however, the inner coating is thinner, which suggests a different drying and hydrolysis behaviour on both sides. Also, the average roughness $R_{\mathrm{a}}$ (Fig. 7c,d) has been decreased for both tube diameters to values of approximately $1 \mathrm{~nm}$, comparable to the value obtained on flat substrates. It is remarkable that the outer coatings show a larger average roughness $R_{\mathrm{a}}$. This possibly indicates that the flow inside the tube is now under better control than outside where turbulences are always difficult to avoid.

The smoother surface of the ATO coatings is confirmed by the morphology as seen from the SEM images (Fig. 3b,d) that show only small cracks. Similar to the results obtained for the roughness, the inner coating looks even smoother than the outer coating. The defects occurring in these images seem not to be an intrinsic problem of the forced flow, as they show both on the
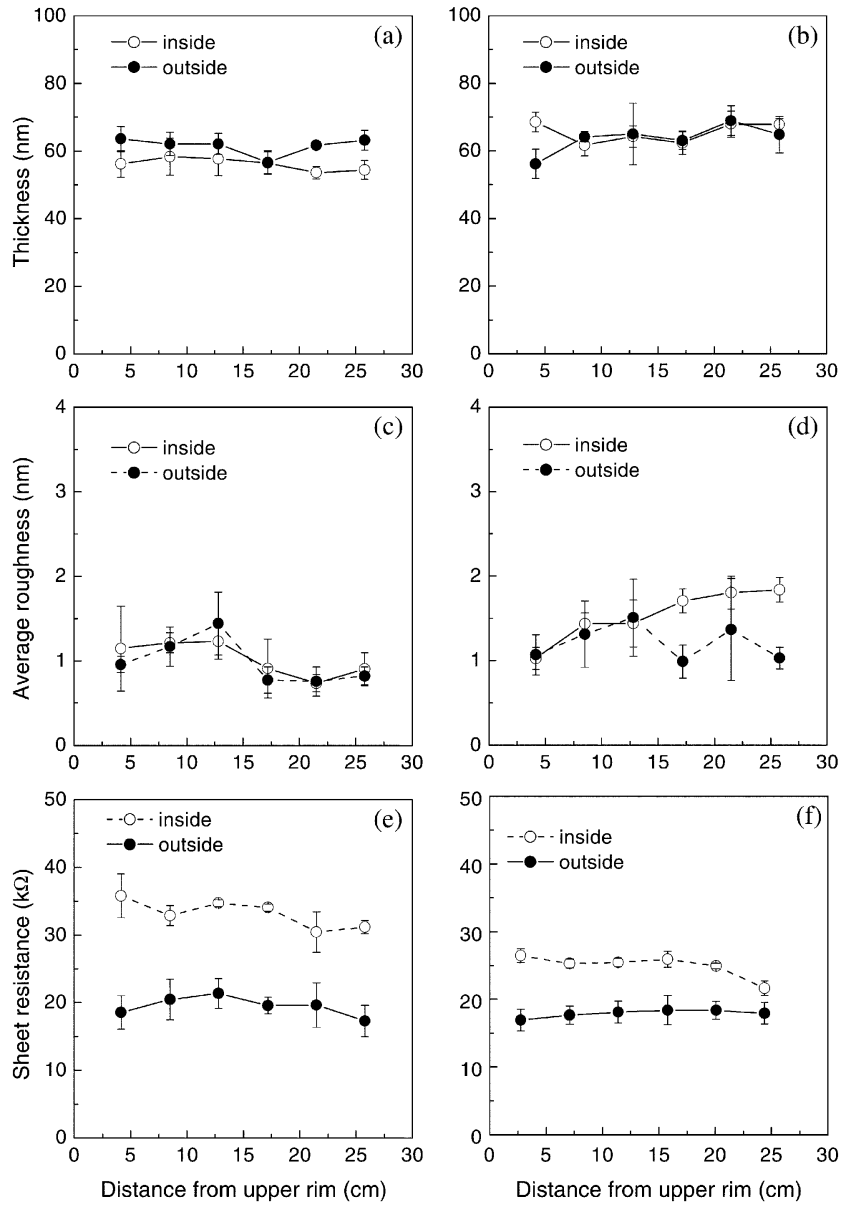

Fig. 7. Thickness $d$, average roughness $R_{\mathrm{a}}$ and sheet resistance $R_{\square}$ of ATO coatings deposited under forced flow on tubes of inner diameters of $11 \mathrm{~mm}$ (left) and $46.4 \mathrm{~mm}$ (right).

(b)

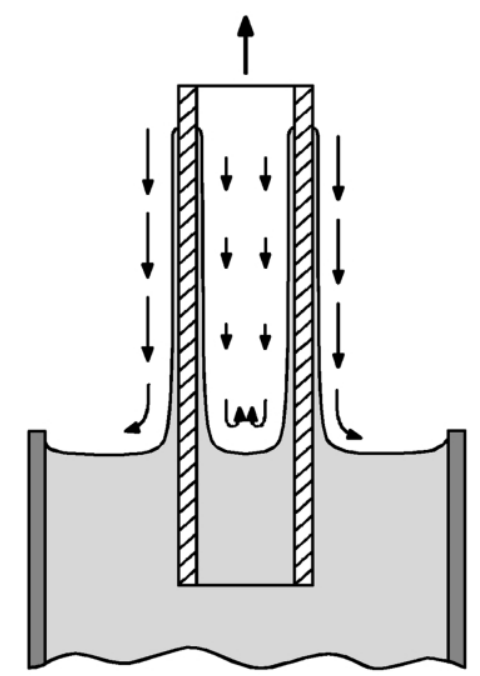

Fig. 6. Schematic illustration of the air flow conditions during conventional dip coating of (a) flat substrates and (b) tubes. 


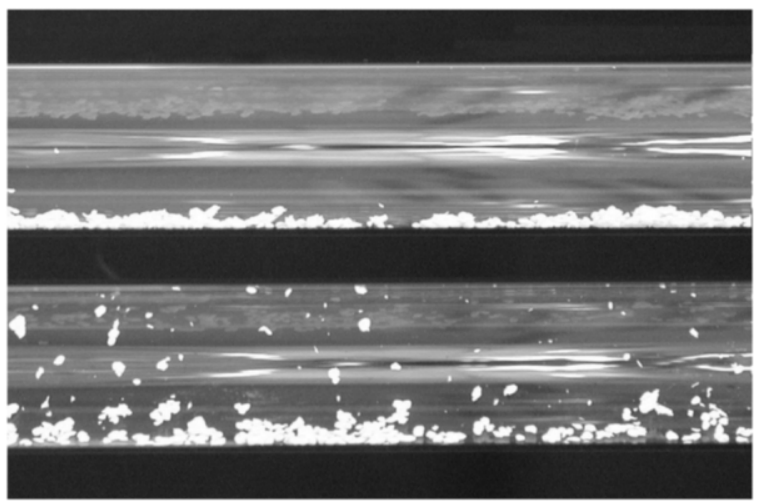

Fig. 8. Photograph of an ATO antistatic-coated (upper) and an uncoated glass tube (lower) with polystyrene beads.

outer and the inner coating, but rather due to a contamination of the coating solution.

The improved uniformity and morphology also favourably influence the electrical properties. The sheet resistance of the ATO coatings (Fig. 7e,f) accordingly is almost uniform with only small deviation at the ends of the tubes. In both cases the outer sheet resistance with values of less than $20 \mathrm{k} \Omega_{\square}$ is significantly lower than inside, especially for the smaller tube diameter with $30-35 \mathrm{k} \Omega_{\square}$. The lower values outside and the fact that the sheet resistance inside is lower for the larger tube suggest that these differences are due to differences in the atmosphere during the heat treatment.

\subsection{Applications}

Transparent conducting sol-gel ATO coatings can be used in areas where only a moderate electronic conductivity is required. Typical applications are, for example, the realization of antistatic properties inside of tubes as demonstrated in Fig. 8, for which a sheet resistance of some 10 to $100 \mathrm{k} \Omega_{\square}$ usually is sufficient. But also electrical heating systems and electrodes can be realized inside tubes. Moreover, the thickness uniformity of the coatings can be used for the preparation of interference systems with other oxide materials like $\mathrm{SiO}_{2}$ and $\mathrm{TiO}_{2}$ (colour filters, antireflective coatings) and to combine them with the antistatic properties of ATO.

\section{Conclusions}

The conventional dip coating of tubes with sol-gel ATO leads to inhomogeneous and non-reproducible coating properties with regards to the thickness, the average roughness and the electrical properties. The main reason for this is the saturation of the inner volume with solvent vapour during the drying of the wet film and the consequently impeded flow conditions inside the tube. A laminar flow usually forms as a result of the descending solvent vapour and is a fundamental process in the sol-gel film formation as it guarantees a permanent renewal of the atmosphere close to the film surface and thus keeps the evaporation rate and the water content constant. In order to realize similar conditions inside the tubes, the dip coating apparatus was modified by placing an exhausting pipe from below into the tube which makes it possible to obtain a forced flow during the entire withdrawal process. The resulting ATO coatings accordingly show a uniform thickness and an extremely low roughness with homogeneous and reproducible electrical properties. Due to the marked uniformity of the coatings the preparation of other types of functional coatings and especially multilayer coatings in optical quality becomes possible even inside tubes. With a slightly modified coating solution a resistance of $(10 \pm 0.5) \mathrm{k} \Omega$ measured over a distance of $22 \mathrm{~cm}$ could be realized reproducibly in a single coating step in tubes with an inner diameter of $11 \mathrm{~mm}$. A further reduction of the resistance can be achieved with multilayer ATO coatings.

\section{References}

[1] H.L. Hartnagel, A.L. Dawar, A.K. Jain, C. Jagadish, Semiconducting Transparent Thin Films, Institute of Physics Publishing, Bristol, 1995.

[2] B.G. Lewis, D.C. Paine, MRS Bulletin 25 (2000) 22.

[3] H.J. Gläser, Large Area Glass Coating, Von Ardenne Anlagentechnik GmbH, Dresden, 2000.

[4] A. Mucha, H. Mai, R. Dietsch, EP Patent No. 0729520 B1, 28 October 1994.

[5] A. Schumacher, G. Frech, G.K. Wolf, Surf. Coat. Technol. 89 (1997) 258.

[6] Y. Yamada, JP Patent No. 63026373 A, 18 July 1986.

[7] S.F. Kistler, P.M. Schweizer, Liquid Film Coating, Chapman and Hall, London, 1997.

[8] Y. Yashiki, Y. Kawamorita, JP Patent No. 60263157 A, 12 June 1984.

[9] Y. Yashiki, H. Moriyama, K. Aoki, JP Patent No. 63302977 A, 2 June 1987.

[10] O. Igawa, JP Patent No. 7104488 A, 31 May 1993.

[11] O. Muller, J. Puetz, Unpublished. 